\title{
Review Article \\ Epigenetic Dynamics: Role of Epimarks and Underlying Machinery in Plants Exposed to Abiotic Stress
}

\author{
Manoj Kumar Dhar, ${ }^{1}$ Parivartan Vishal, ${ }^{1}$ Rahul Sharma, ${ }^{1}$ and Sanjana Kaul ${ }^{2}$ \\ ${ }^{1}$ Plant Genomics Laboratory, School of Biotechnology, University of Jammu, Jammu 180006, India \\ ${ }^{2}$ School of Biotechnology, University of Jammu, Jammu 180006, India \\ Correspondence should be addressed to Manoj Kumar Dhar; manojkdhar@rediffmail.com
}

Received 16 May 2014; Revised 28 July 2014; Accepted 7 August 2014; Published 18 September 2014

Academic Editor: Henry Heng

Copyright (C) 2014 Manoj Kumar Dhar et al. This is an open access article distributed under the Creative Commons Attribution License, which permits unrestricted use, distribution, and reproduction in any medium, provided the original work is properly cited.

\begin{abstract}
Abiotic stress induces several changes in plants at physiological and molecular level. Plants have evolved regulatory mechanisms guided towards establishment of stress tolerance in which epigenetic modifications play a pivotal role. We provide examples of gene expression changes that are brought about by conversion of active chromatin to silent heterochromatin and vice versa. Methylation of CG sites and specific modification of histone tail determine whether a particular locus is transcriptionally active or silent. We present a lucid review of epigenetic machinery and epigenetic alterations involving DNA methylation, histone tail modifications, chromatin remodeling, and RNA directed epigenetic changes.
\end{abstract}

\section{Introduction}

Stress is inevitable in the life cycle of living organisms. Being sessile, plants are more prone to the deleterious effects of environmental stress. Depending upon whether the factors involved are living or nonliving, environmental stress can be categorized as biotic (plant pathogens, etc.) or abiotic stress (drought, salinity, chilling, etc.). Stressful conditions generally do not occur as isolated events but as crosstalk of multiple stresses. Therefore, plants have developed complex mechanisms to survive under these challenging conditions. Tolerance, avoidance, and resistance are three major strategies followed by plants to counter the recurring biotic and abiotic stresses. These mechanisms involve genes associated with several interconnected pathways which lead them towards better stress tolerance [1]. Plants resort to various modifications in their morphological traits, physiology, and so forth in response to stress.

Depending upon whether the stress is permanent or transitory, plants respond through various short term as well as long term strategies. Short term strategies include alteration in the plant homeostasis. Restoration of cellular homeostasis reduces stress injury by eliminating consequences of stress which leads to development of stress tolerance. Long-term strategies include transgenerational changes involving development of heritable gene expression changes. This comprises creation of new epigenetic marks while erasing old ones and increasing the expression of some genes while silencing some other genes. Severe and prolonged stress can lead to genome alterations which may sometimes contribute towards better adaptation [2]. The basic information guiding the behavior of plant lies in the DNA sequence and alterations in DNA sequence by mutation or genetic recombination lead to new alleles which may confer enhanced stress tolerance to the plant. However, the rate of formation of new gene combinations is too slow in comparison to the occurrence of different stresses in the environment [3]. Therefore, the survival of plant in these conditions depends largely on the regulation of various stress responsive genes, that is, epigenetic mechanisms. Epigenetic changes include any heritable change in an organism which does not involve change in the DNA sequence. Rather, it involves addition or deletion of epimarks (methylation, etc.) on DNA, posttranslational modifications on histone tails (acetylation, methylation, sumoylation, etc.), and RNA interference.

Histone modifications and alterations in DNA methylation are together referred to as epigenetic regulation but only those changes which are either mitotically or meiotically 
heritable are truly epigenetic [4]. These alterations affect gene expression by chromatin remodeling which involves change in chromatin state of the chromosome, that is, euchromatin or heterochromatin. For instance, in order to increase the expression of a particular gene, its promoter sequence must be exposed so that transcription factor and RNA polymerase could bind to the underlying upstream DNA and carryout transcription of the gene. In order to expose the DNA for efficient transcription, nucleosome complex must be untangled. Conversely, for shutting off gene expression, DNA methylation has to be reestablished, followed by packaging of the DNA stretch covering that particular gene by the histone components of the nucleosome complex.

\section{Epigenetic Alterations and Requisite Machinery}

2.1. DNA Methylation. Methylation is the only covalent modification that has been identified on DNA till date [5]. It involves addition of methyl group $\left(-\mathrm{CH}_{3}\right)$ at fifth carbon in the cytosine ring of the DNA molecule at CpG, CpNpG (symmetric), or $\mathrm{CpNpN}$ (asymmetric) sites (where $\mathrm{N}$ is A, C, or T).

Mechanism of DNA methylation is governed by two types of enzymes:

(i) methyltransferases;

(ii) demethylases.

Two major enzymatic activities regulate cytosine methylation in plants which involve de novo establishment of methylation on DNA and maintenance of the already methylated DNA. The de novo methylation is a process by which previously unmethylated cytosine residues are methylated, leading to the formation of new methylation patterns. Maintenance methylation is the process of maintenance of preexisting methylation patterns after DNA replication [6]. MET1 (DNA methyltransferase 1) and CMT (chromomethylase) are responsible for maintenance of $\mathrm{CG}$ and $\mathrm{CNG}$ methylation, respectively [7]. De novo methylation is established by DRM2 (domains rearranged methyltransferase 2) in the new DNA sequences generated after DNA replication. DNA gycosylases (ROS1, DML2, DML3, and DME) catalyze the removal of methyl group from cytosine residue [8].

Genome-wide analysis of DNA methylation in Arabidopsis thaliana revealed the methylation status of its genome as 24\% CG, 6.7\% CNG, and 1.7\% CNN methylation [9]. CpNpG and $\mathrm{CpNpN}$ methylation changes mediated by CMT3 and DRM2 have been reported to regulate transposons and repeat regions through chromatin remodelling during exposure to stress $[10,11]$.

DNA methylation is distributed in the plant genome including heterochromatic and euchromatic regions [12]. The heterochromatic regions, densely packed with transposable elements and other repetitive sequences, are highly methylated whereas euchromatic regions, containing genes and nonrepetitive intergenic regions, show comparatively lesser cytosine methylation. Interestingly, transposons are methylated along their entire length in contrast to genes which are often methylated away from the start and termination sites.
Within the euchromatic region, pseudogenes and transcriptionally inactive genes show higher levels of methylation than actively expressing genes [13]. Expressed genes are methylated in the transcribed region (gene-body methylation) [14]. Gene body methylation exhibits a parabolic relationship with transcription level in rice and Arabidopsis. Both the least expressed and the highly expressed genes are least prone to methylation whereas moderately expressed genes are most likely to be methylated at gene body $[15,16]$. Genic regions do not contain non-CG methylation while transposons and repeats abundantly possess $\mathrm{CpNpG}$ or $\mathrm{CpNpN}$ methylation. Methylation at $5^{\prime}$ portion (promoter plus some transcribed region) and $3^{\prime}$ portion inhibits gene expression.

Stress can cause hypermethylation or hypomethylation of DNA. In maize roots, cold induced expression of ZmMI1 was accompanied with a decrease in DNA methylation which did not revert to basal level even after 7 days of recovery. In tobacco, aluminium, salt, cold, and paraquat stresses induced DNA demethylation at CG nucleotides in the coding sequence of NtGPDL gene (glycerophosphodiesterase-like protein) [17]. Heavy metal stress is known to induce hypomethylation at specific sites in the genome of both the metalsensitive Trifolium repens L. and metal-tolerant Cannabis sativa L. [18]. DNA hypermethylation at CG but not CNG at two heterochromatic loci was induced in cell suspension culture of tobacco by osmotic stress [19]. Drought-induced hypermethylation has been proposed to play a primary and direct role in reducing the metabolic activity in pea root tips after 72-hour water deficit [20, 21]. Suji and Joel [22] reported drought induced hypermethylation and hypomethylation in drought tolerant and drought susceptible varieties of rice, respectively. Stress induced hypermethylation of satellite DNA was associated with a switch in photosynthesis mode from C3 to CAM in Mesembryanthemum crystallinum L., a facultative halophyte [23].

Promoter demethylation is known to abolish constitutive gene silencing established because of hypermethylation of $X a 21 G$ gene, thereby conferring disease resistance in rice [24]. Changed methylation level in maize exposed to osmotic and salt stress helps in stress acclimation [25]. Stressful environment produces transgenerational epigenetic modifications leading to enhanced stress adaptability in future progenies [26]. Nonstressed progenies of stressed rice plants carrying modified methylation patterns acquired from the parent exhibit enhanced stress tolerance [27].

Transposons and other repeats constitute large part of the plant genome and cytosine methylation is chiefly targeted towards transposon silencing $[28,29]$. CG and non-CG methylation contribute towards transposon immobilization. In plants, non-CG methylation is proposed to have evolved as an epigenetic tag committed to transposon control [30]. A close relationship between methylation and low temperature dependent transposition (LTDT) has been reported, where low temperature caused reduction in methylation level opposed to hypermethylation resulting from higher temperature in Antirrhinum majus [31]. Transposon methylation changes which control transposition activity of transposons are also reported to spread silencing signal to neighboring 


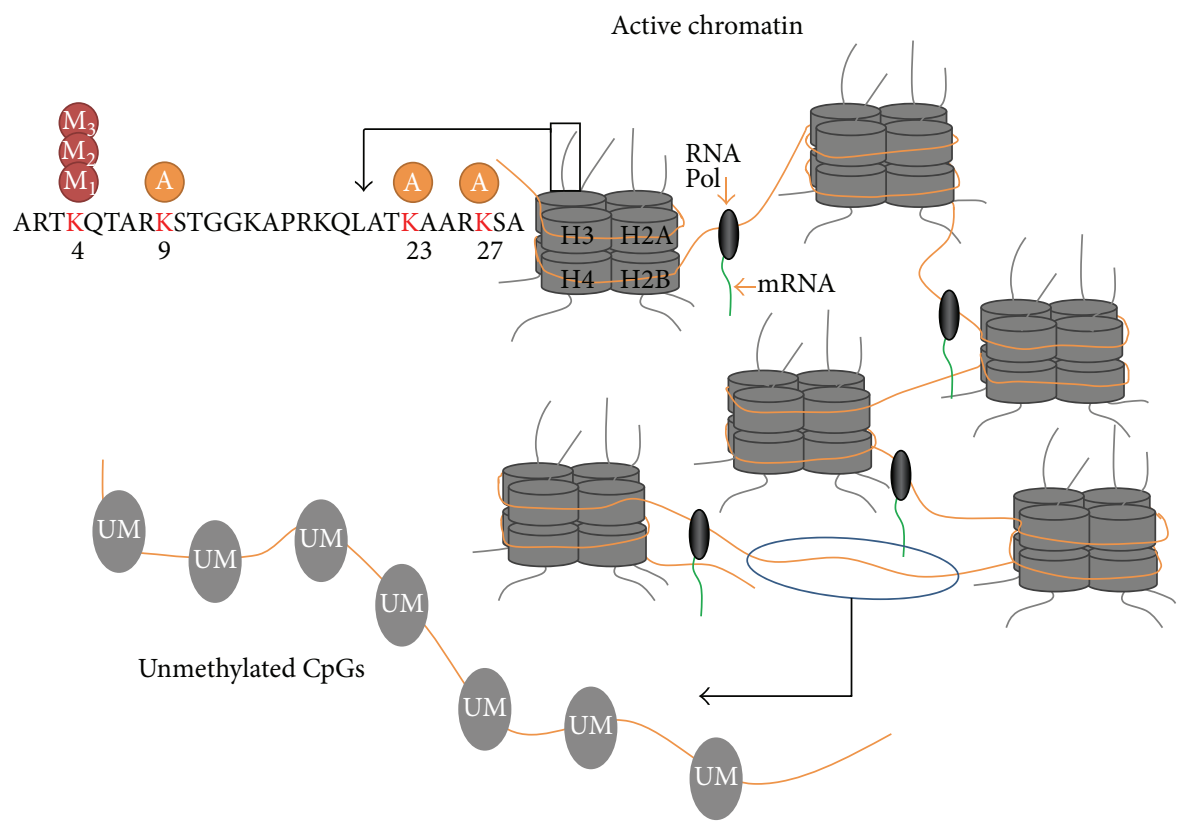

FIGURE 1: Epigenetic marks associated with transcriptionally active chromatin. Trimethylation at K4 and acetylation at K9, K23, and K27 of H3 and unmethylated CGs signify active chromatin.

genes. Tos17 methylation spreads to upstream ABC-transporter-like gene [32].

2.2. Histone Code. Histones are very crucial for packaging of DNA. DNA folds around histone octamer $(\mathrm{H} 2 \mathrm{~A}, \mathrm{H} 2 \mathrm{~B}$, $\mathrm{H} 3$, and $\mathrm{H} 4$ ) to form nucleosome, which is the basic unit of chromatin. The organization of chromatin is critical for transcription and many other cellular processes like replication, repair, recombination, and so forth. This organization is directly influenced by posttranslational modifications in the histone tails protruding out of their amino terminal. The histone tails are reported to interact with negative charge on the DNA and other associated proteins [33]. These interactions are altered by certain posttranslational modifications targeted towards specific amino acid residues and depending upon the posttranslational modification on histone tail, the integrity of nucleosome in that region is determined. These modifications include methylation, acetylation, phosphorylation, ubiquitination, biotinylation, and sumoylation at specific amino acid residues [34]. A combination of site-specific posttranslational modifications on different residues of histone tail constitutes "histone code." Each modification signifies a particular chromatin state and regulates transcriptional activity in combination with different external and internal signals.

2.3. Modifying Enzymes. Histone acetyltransferases (HATs) carry out acetylation of histone tails and are associated with gene activation. HATs transfer acetyl group to $\varepsilon$-amino group of lysine residues in the $\mathrm{N}$-terminal extensions of nucleosomal core histones.

Lysine $(\mathrm{K})$ bears positive charge and the transfer of acetyl group neutralizes this positive charge. This reduces the affinity of nucleosome complex for DNA leading to relaxed chromatin state and subsequent transcriptional activation. About
15 HATs have been reported in Arabidopsis, which belong to three families: GNAT/MYST, CBP, and TF II250 [35]. HATs interact with TFs and activate stress responsive genes which regulate stress tolerance. SAGA (HAT) interacts with ADA1 (TF) and the SAGA/ADA1 complex interacts with CBF1 which recruits this complex to activate downstream genes for better cold tolerance [36].

Histone deacetylases (HDACs) are responsible for deacetylation, that is, removal of acetyl group from histones, leading to condensed chromatin state and thereby causing gene silencing [37]. HDACs are further divided into three families [38], namely, (a) RPD3 family, (b) SIR2 family, and (c) HD2 family. Both HATs and HDACs affect the expression of developmental and stress responsive genes.

HMTs (histone methyl transferases) and HDMs (histone demethylases) are responsible for methylation and demethylation of histone tails, respectively.

Histone methylation occurs at lysine and arginine amino acids. All lysine methylations are carried out by HKMTs (histone lysine methyltransferases) containing SET domain [39]. They are classified in to five classes, Class I to Class V (Table 1).

Histone Demethylases (HDMs). There are two types of demethylases which carry out oxidative demethylation of histones (Table 2).

\subsection{Histone Modifications}

2.4.1. H3K Acetylation. Acetylation of lysine residues is very flexible and plays a vital role in the life cycle of plants [7]. Active chromatin is marked by $\mathrm{H} 3$ acetylation resulting in relaxation of chromatin state which facilitates the transit of RNA polymerase [42] (Figure 1). Histone lysine acetylation 


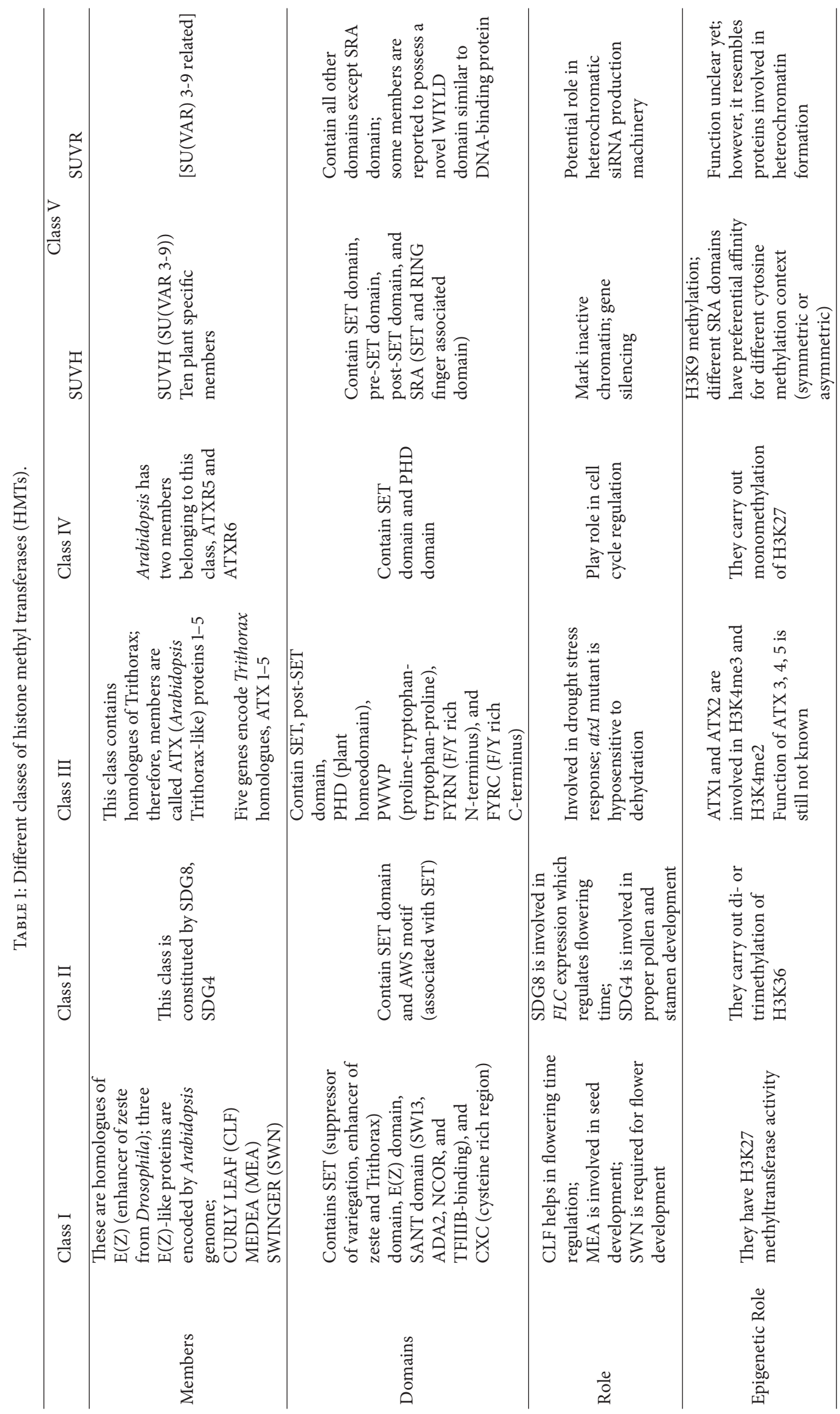


TABle 2: Types of Histone demethylases (HDMs).

\begin{tabular}{ll}
\hline Lysine specific demethylase 1 (KDM/LSD1) & Jumonji C domain containing proteins (JmjC) \\
\hline Require flavin as cofactor & Require Fe(II) and $\alpha$-ketoglutarate as cofactors \\
\hline $\begin{array}{l}\text { Remove methyl group from mono- and dimethylated } \\
\text { lysines on histone tails. }\end{array}$ & Remove methylation from mono-, di-, and trimethylated lysines \\
\hline & $\begin{array}{l}\text { Twenty one JmjC-domain proteins are reported in Arabidopsis which are } \\
\text { classified into 5 subfamilies: }\end{array}$ \\
Four KDM/LSD1 demethylases in Arabidopsis: & KDM5/JARID1 group \\
Flowering locus D (FLD) & KDM4/JHDM3 group \\
LSD1 like (LDL1) & KDM3/JHDM2 group \\
LDL2 & JMJD6 group \\
LDL3 & JmjC-domain only \\
& KDM4/JHDM3 proteins along with ELF6/JMJ11 (early flowering 6) and \\
& REF/JMJ12 (relative of early flowering) control flowering time (Yu et al. \\
FLD, LDL1, and LDL2 are involved in flower induction & 2008) [40], \\
in Arabidopsis through flc repression & bonsai methylation), protects active genes from ectopic H3K9me2 and \\
& CNG DNA methylation [41] \\
\end{tabular}

rearrangement has been reported to be associated with flowering [43] and cold stress tolerance [44]. HDA6 and HDA19 expression is induced by stress and affects local chromatin structure. HDA6 has been reported to be responsible for deacetylation of histones in response to biotic and abiotic stress induced by jasmonic acid and ethylene in Arabidopsis. Overexpression of AtHD2C in transgenic Arabidopsis results in increased expression of ABA-responsive genes (LEA) leading to improved salt and drought stress tolerance [45]. Hos15 protein interacts with $\mathrm{H} 4$ and carries out $\mathrm{H} 4$ deacetylation thereby regulating stress tolerance in Arabidopsis [44].

$\mathrm{H} 3 \mathrm{~K} 4 \mathrm{me} 3$ and $\mathrm{H} 3 \mathrm{~K} 9$ acetylation on promoter region and $\mathrm{H} 3 \mathrm{~K} 23$ acetylation and $\mathrm{H} 3 \mathrm{~K} 27$ acetylation on coding region affect gene expression of stress responsive genes [35]. Four drought responsive genes (RD29A, RD29B, RD20, and RAP2.4) have been reported to exhibit enrichment of $\mathrm{H} 3 \mathrm{~K} 4 \mathrm{me} 3$ and $\mathrm{H} 3 \mathrm{~K} 9$ acetylation and activation in response to drought stress. Moreover, there is a gradual decrease of nucleosomal density on RD20 and RAP2.4 genes under drought stress [35].

2.4.2. H3K Methylation. H3 lysine methylation is the most abundant histone modification. Lysine can be mono-, di-, or trimethylated. H3K9 methylation is a characteristic of heterochromatin and signifies silencing of the locus [46] (Figure 2). Despite this, the loss of this mark does not always represent the activation of the region suggesting the involvement of other important factors also [47]. H3K27me3 is a major chromatin silencing modification found associated with $5^{\prime}$ region of thousands of genes in Arabidopsis [48]. On the other hand, $\mathrm{H} 3 \mathrm{~K} 9 \mathrm{me} 3$ is a repressive chromatin modification associated with gene coding region [49]. H3K9me2 is localized in heterochromatic region, transposons, pseudogenes, and repeats [50]. All H3K4me marks are associated with active chromatin. H3K4me3 and $\mathrm{H} 3 \mathrm{~K} 4 \mathrm{me} 2$ are associated with promoter and $5^{\prime}$ part of the transcribed gene while $\mathrm{H} 3 \mathrm{~K} 4 \mathrm{mel}$ covers terminal part $\left(3^{\prime}\right)$ of the gene [51]. Silent chromatin (heterochromatin) bears $\mathrm{H} 3 \mathrm{~K} 9$ me which recruits other proteins such as heterochromatin protein 1 (LHP1). These bind to methylated $\mathrm{H} 3 \mathrm{~K} 9$ and help in the propagation of heterochromatin to adjacent region of the chromosome [52].

Drought-inducible linker histone variant (H1S) in tomato is responsible for negative regulation of stomatal closure [53]. $\mathrm{H} 3 \mathrm{~K} 4 \mathrm{me} 3$ and $\mathrm{H} 3$ acetylation was found to be induced in alcohol dehydrogenase 1 (ADH1) and pyruvate decarboxylase 1 (PDC1) genes. These changes were reverted back on withdrawal of submergence stress [54].

$\mathrm{H} 3 \mathrm{~K} 4 \mathrm{me}$ marks on nucleosomes of stress-inducible genes have been reported to be associated with the activation of chromatin in response to dehydration [55]. H3Kme marks are reported to be present in $90 \%$ of annotated Arabidopsis genes wherein abundance of $\mathrm{H} 3 \mathrm{~K} 4 \mathrm{me} 3$ mark is directly related to level of transcriptional activity of the drought responsive genes. Increase in $\mathrm{H} 3$ phosphorylation and $\mathrm{H} 3$ and $\mathrm{H} 4$ acetylation in response to abiotic stresses have been found in tobacco and Arabidopsis [56].

2.4.3. Other Histone Modifications. In Arabidopsis an arginine methyltransferase SKB1 (also known as protein arginine methyl transferase5 PRMT5) is involved in abiotic stress response. SKB1 is normally associated with chromatin and increases level of arginine trimethylation on H4 (H4Rme2) so as to repress gene expression. With the onset of salt stress, SKB1 dissociates from chromatin and results in induction of stress responsive genes. $s k b 1$ mutant is hypersensitive to salt stress [57].

2.5. RNA Directed DNA Methylation (RdDM). Abiotic stress has been reported to modulate the expression of several hundred genes, and depending upon their roles they are either upregulated or downregulated. Apart from the regulatory control at the level of transcription, the posttranscriptional regulation is also important for regulation of gene expression. This is achieved by RNA binding proteins (RBPs) which bind 


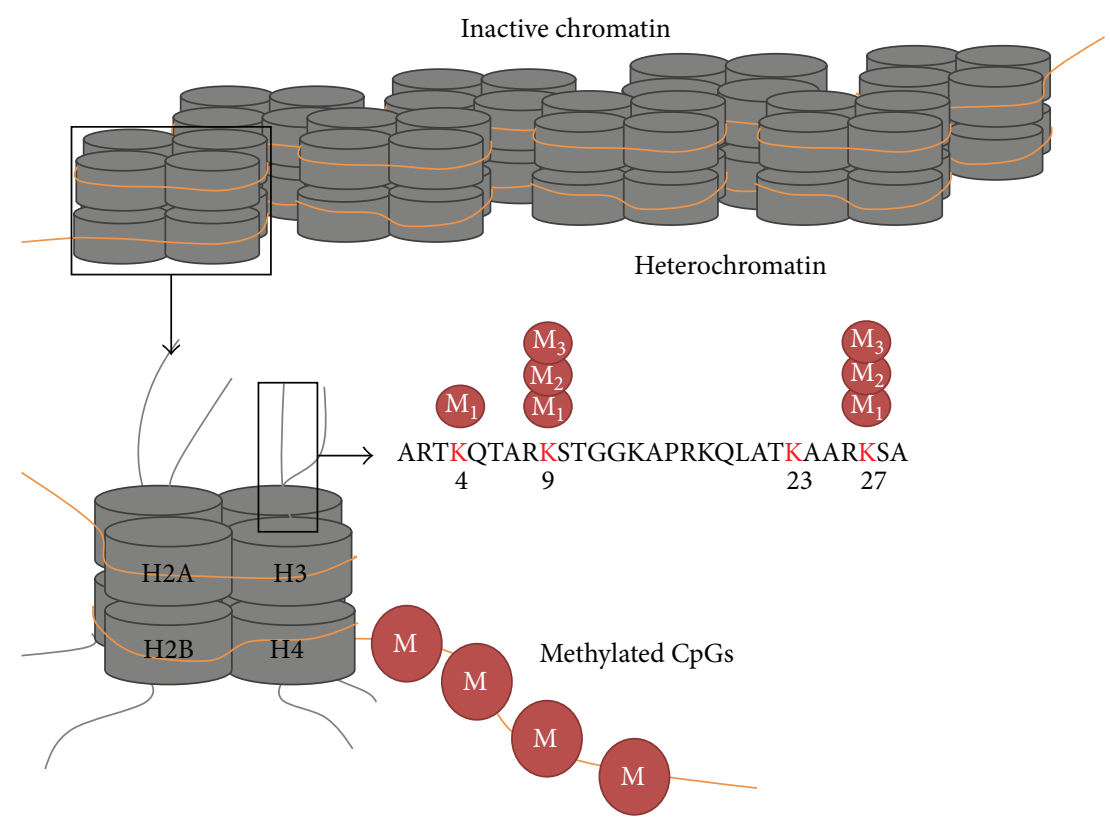

FIGURE 2: Epigenetic marks associated with transcriptionally inactive chromatin. Methylation at K4, K9, and K27 of H3 and methylated CGs indicate silent chromatin.

to UTRs of mRNAs and control their stability, localization, or translation. In addition to this, small RNAs (microRNAs and small interfering RNAs) play a vital role in gene regulation [58]. RNAi machinery is necessary for the maintenance of heterochromatin and silencing of repetitive DNA, transposons, and so forth [59]. RNA directed DNA methylation ( $\mathrm{RdDM})$ is known to be regulated by temperature. Virusinduced gene silencing is promoted at low temperature and delayed by high temperature [60]. Though promoters are also methylated de novo, TEs and other repetitive DNA elements are effectively silenced by this mechanism [61].

2.5.1. miRNA. MicroRNAs are short (20-24 nucleotide), endogenous RNAs, processed by Dicer-like enzyme from longer transcripts, which are not translated into proteins [58]. Plant miRNAs genes have been found away from protein coding regions of the genome and are expressed by their own transcription unit [62].

Role of miRNAs in gene regulation vis-à-vis abiotic stress has been best studied by Sunkar et al. [58]. Genes which are negative regulators of stress tolerance (i.e., repress stress responsive genes) are downregulated during stress by upregulation of microRNAs targeting these genes. On the other hand, miRNA downregulation under stress results in accumulation of mRNAs of those genes which act as positive regulators of stress tolerance [58].

Overexpression of miR396 in Arabidopsis and rice plants resulted in reduced tolerance to salt and alkali stress [63]. Sequence analysis of small RNA library of stress treated Arabidopsis thaliana showed that miR393 was the most abundantly expressed miRNA and its level increased by a variety of stresses like cold, salt, ABA, and dehydration. Some stress-specific expression of miRNAs was also observed; for instance, miR319c is upregulated by cold but not by ABA salt or dehydration [64]. Cold stress resulted in differential expression of a number of miRNAs including miR319 in rice and Brachypodium $[65,66]$. On oxidative stress, miR398 is transcriptionally downregulated, therefore, leading to the accumulation of CSD1 and CSD2 mRNAs which are crucial for plant stress resistance. mRNAs of these two genes do not accumulate under normal conditions because of miR398guided cleavage [67]. miR160 and miR164 along with their target genes have been reported to play an important role in the regulation of root growth in Arabidopsis during drought stress. Overexpression of miR160 led to agravitropic roots and increase in the number of lateral roots [68]. Manipulation of miRNA-guided gene regulation can help development of stress-resistant plants [69].

2.5.2. siRNA. Small interfering RNAs (siRNAs), 20-24 nucleotides in length, are known to play an important role in a range of processes, such as heterochromatin formation, transposon silencing, transgene silencing, posttranscriptional regulation of mRNAs, and defense against viruses. Processing of long dsRNAs generated from natural cis-antisense gene pairs, repetitive DNA, or noncoding transcripts by Dicer-like enzymes generate small interfering RNAs [58].

After processing, one of the strands of the duplex serves as guide strand and is loaded onto RITS (RNA-induced transcriptional silencing complex). The complex binds to siRNA by PAZ domain of AGO4 protein and is directed to the homologous DNA sequence for gene silencing at transcriptional level (TGS). AGO4 is associated with Pol V which synthesizes transcripts that interact with siRNAs to induce DNA methylation at the targeted site by DRM2 (de novo methyltransferase) [10]. Nascent RNAs bind to the target DNA sequences and recruit histone methylases to add methyl group to lysine residues at 9 or 27 position of $\mathrm{H} 3$ histone 
tails. This leads to recruitment of DNA methylases which transfer methyl group to DNA ultimately leading to gene silencing and heterochromatin formation [70, 71]. The methylated DNA serves as template for Pol IV. Pol IV transcribes the methylated DNA and its downstream sequence to produce aberrant RNA transcripts which subsequently generates dsRNA by the activity RDR2 (RNA-dependant RNA polymerase 2). These RDR2 synthesized dsRNAs act as precursor for secondary siRNA which help in spreading methylation to adjacent sequences [10].

One of the possible mechanisms of regulation of plant stress response is the inhibition of siRNA biogenesis. Dcl2 and $\mathrm{Dcl} 3$ mutants having weakened transactivation activity of siRNA biogenesis were more sensitive to MMS (methylmethane sulfonate) which causes genotoxic stress [72].

An excellent example of regulation of stress tolerance is that of genes involved in proline catabolism in Arabidopsis. SR05 is induced by salt stress. SR05 mRNA is complementary to $\mathrm{P} 5 \mathrm{CDH}$ mRNA (P5CDH protein is an important enzyme for proline breakdown) and they together generate a dsRNA which is acted upon by siRNA biogenesis pathway factors (DCL2, RDR6, SGS3, and NRPD1A) to produce 24nt-siRNA. This nat-siRNA guides the cleavage of P5CDH mRNAs leading to proline accumulation and better salt tolerance. SR05 mutants exhibit hypersensitivity to salt stress [73].

2.6. Chromatin Remodelling Factors (CRMs). Chromatin remodeling factors are multisubunit protein complexes which modify chromatin structure by influencing histone-DNA interactions in order to assemble, destabilize, or displace nucleosomes using ATP derived energy [74]. High CRM concentration results in histone octamer transfer to another DNA molecule. At moderate concentration, they facilitate sliding of the octamer position leading to altered gap between adjacent nucleosomes to facilitate access of TFs, restriction enzymes, and so forth.

ATP dependent chromatin remodeling factors can be grouped into three categories:

\section{(1) SWF/SNF ATPases;}

(2) ISWI (Imitation Switch) ATPases;

(3) CHD (chromodomain and helicase-like domain) ATPases.

SWF1/SNF complex was originally identified for defects in mating type switching (SW1) and sucrose fermentation (sucrose nonfermenting) [75]. ATCHR12, a SNF2/Brahmatype chromatin-remodelling protein, plays an important role in temporary growth arrest of normally active primary buds in Arabidopsis thaliana exposed to stress [76]. SW13 subunit of SW1/SNF complex has been recently reported to act as a positive regulator in ABA-mediated inhibition of seed germination and growth by interacting with a negative regulator, $\mathrm{HAB} 1$ (Hypersensitive to $\mathrm{ABA1}$ ), to increase the expression of RAB18 and RD29B [77]. Another chromatin remodeling factor PICKLE (PKL) helps in maintaining AB13 and $\mathrm{AB} 15$ chromatin in a repressed state during germination indicated by reduced $\mathrm{H} 3 \mathrm{~K} 9$ and $\mathrm{H} 3 \mathrm{~K} 27$ methylation level in pkl mutant seeds when treated with ABA [78].
Histone chaperons are known to carry out nucleosome assembly and disassembly by deposition or expulsion of histones, respectively. NAP1 (nucleosome assembly protein 1) is known to function as chaperon for $\mathrm{H} 2 \mathrm{~A}$ and $\mathrm{H} 2 \mathrm{~B}$ histones in Arabidopsis [79]. AtNAPs are reported to be positive regulators of ABA signaling pathway [80]. MSI1, a WD40 repeat protein acting as a subunit for many protein complexes (like chromatin assembly factor 1 and Polycomb group protein complexes), is involved in chromatin assembly and plays the role of a negative regulator in drought stress response in Arabidopsis [81]. Plants with highly reduced MSI1 levels exhibit enhanced level of ABA-responsive gene transcripts [82]. COR (Cold regulated) genes containing C/DRE (C-repeat/dehydration responsive element) are also regulated negatively by MSI1-like protein MSI4/FVE [83].

\section{Conclusion}

Stress-induced epigenetic changes in the form of DNA methylation, histone tail modifications, and RNA directed DNA methylation are governed by a complex phenomenon involving myriad factors interacting among themselves. These changes in epigenetic marks modulate transcription of stressresponsive genes leading to the formation of heritable epialleles which subsequently enable plant to withstand stress. There is a need for the identification of such epialleles along with comprehensive understanding of the fundamental epigenetic mechanisms. Importantly, it is necessary to study epigenetic heterogeneity (a key aspect of epigenetic dynamics) both at epialleles level and whole genome level [84]. Complete knowledge of these mechanisms would lay a platform for the researchers to devise better strategies for crop improvement like exploitation of small RNAs for the manipulation of epialleles.

\section{Conflict of Interests}

The authors declare that there is no conflict of interests regarding the publication of this paper.

\section{Acknowledgments}

The authors are grateful to the Department of Biotechnology, Government of India, for the financial support. Thanks are also due to the Bioinformatics Centre at School of Biotechnology, University of Jammu.

\section{References}

[1] S. Mahajan and N. Tuteja, "Cold, salinity and drought stresses: an overview," Archives of Biochemistry and Biophysics, vol. 444, no. 2, pp. 139-158, 2005.

[2] S. D. Horne, S. K. Chowdhury, and H. H. Q. Heng, "Stress, genomic adaptation, and the evolutionary trade-off," Frontiers in Genetics, vol. 5, article 92, 2014.

[3] H. Peng and J. Zhang, "Plant genomic DNA methylation in response to stresses: potential applications and challenges in plant breeding," Progress in Natural Science, vol. 19, no. 9, pp. 1037-1045, 2009. 
[4] V. Chinnusamy and J. K. Zhu, "Epigenetic regulation of stress responses in plants," Current Opinion in Plant Biology, vol. 12, no. 2, pp. 133-139, 2009.

[5] M. Tariq and J. Paszkowski, "DNA and histone methylation in plants," Trends in Genetics, vol. 20, no. 6, pp. 244-251, 2004.

[6] T. Chen and E. Li, "Structure and function of eukaryotic DNA methyltransferases," Current Topics in Developmental Biology, vol. 60, pp. 55-89, 2004.

[7] M. Chen, S. Lv, and Y. Meng, "Epigenetic performers in plants," Development Growth and Differentiation, vol. 52, no. 6, pp. 555566, 2010.

[8] X. Cao, N. M. Springer, M. G. Muszynski, R. L. Phillips, S. Kaeppler, and S. E. Jacobsen, "Conserved plant genes with similarity to mammalian de novo DNA methyltransferases," Proceedings of the National Academy of Sciences of the United States of America, vol. 97, no. 9, pp. 4979-4984, 2000.

[9] J. A. Law and S. E. Jacobsen, "Establishing, maintaining and modifying DNA methylation patterns in plants and animals," Nature Reviews Genetics, vol. 11, no. 3, pp. 204-220, 2010.

[10] M. Matzke, T. Kanno, L. Daxinger, B. Huettel, and A. J. M. Matzke, "RNA-mediated chromatin-based silencing in plants," Current Opinion in Cell Biology, vol. 21, no. 3, pp. 367-376, 2009.

[11] I. J. Furner and M. Matzke, "Methylation and demethylation of the Arabidopsis genome," Current Opinion in Plant Biology, vol. 14, no. 2, pp. 137-141, 2011.

[12] M. Gehring and S. Henikoff, "DNA methylation dynamics in plant genomes," Biochimica et Biophysica Acta-Gene Structure and Expression, vol. 1769, no. 5-6, pp. 276-286, 2007.

[13] I. Ahmed, A. Sarazin, C. Bowler, V. Colot, and H. Quesneville, "Genome-wide evidence for local DNA methylation spreading from small RNA-targeted sequences in Arabidopsis," Nucleic Acids Research, vol. 39, no. 16, pp. 6919-6931, 2011.

[14] S. J. Cokus, S. Feng, X. Zhang et al., "Shotgun bisulphite sequencing of the Arabidopsis genome reveals DNA methylation patterning," Nature, vol. 452, no. 7184, pp. 215-219, 2008.

[15] D. Zilberman, M. Gehring, R. K. Tran, T. Ballinger, and S. Henikoff, "Genome-wide analysis of Arabidopsis thaliana DNA methylation uncovers an interdependence between methylation and transcription," Nature Genetics, vol. 39, no. 1, pp. 61-69, 2007.

[16] A. Zemach, I. E. McDaniel, P. Silva, and D. Zilberman, "Genome-wide evolutionary analysis of eukaryotic DNA methylation," Science, vol. 328, no. 5980, pp. 916-919, 2010.

[17] C.-S. Choi and H. Sano, "Abiotic-stress induces demethylation and transcriptional activation of a gene encoding a glycerophosphodiesteraselike protein in tobacco plants," Molecular Genetics and Genomics, vol. 277, no. 5, pp. 589-600, 2007.

[18] R. Aina, S. Sgorbati, A. Santagostino, M. Labra, A. Ghiani, and S. Citterio, "Specific hypomethylation of DNA is induced by heavy metals in white clover and industrial hemp," Physiologia Plantarum, vol. 121, no. 3, pp. 472-480, 2004.

[19] A. Kovařik, B. Koukalová, M. Bezděk, and Z. Opatrný, "Hypermethylation of tobacco heterochromatic loci in response to osmotic stress," Theoretical and Applied Genetics, vol. 95, no. 1-2, pp. 301-306, 1997.

[20] M. Bracale, M. Levi, C. Savini, W. Dicorato, and M. G. Galli, "Water deficit in pea root tips: effects on the cell cycle and on the production of dehydrin-like proteins," Annals of Botany, vol. 79, no. 6, pp. 593-600, 1997.

[21] M. Labra, A. Ghiani, S. Citterio et al., "Analysis of cytosine methylation pattern in response to water deficit in pea root tips," Plant Biology, vol. 4, no. 6, pp. 694-699, 2002.
[22] K. K. Suji and A. J. Joel, "An epigenetic change in rice cultivars under water stress conditions," Electronic Journal of Plant Breeding, vol. 1, no. 4, pp. 1142-1143, 2010.

[23] O. V. Dyachenko, N. S. Zakharchenko, T. V. Shevchuk, H. J. Bohnert, J. C. Cushman, and Y. I. Buryanov, "Effect of hypermethylation of CCWGG sequences in DNA of Mesembryanthemum crystallinum plants on their adaptation to salt stress," Biochemistry (Moscow), vol. 71, no. 4, pp. 461-465, 2006.

[24] K. Akimoto, H. Katakami, H. J. Kim et al., "Epigenetic inheritance in rice plants," Annals of Botany, vol. 100, no. 2, pp. 205217, 2007.

[25] M. Tan, "Analysis of DNA methylation of maize in response to osmotic and salt stress based on methylation-sensitive amplified polymorphism," Plant Physiology and Biochemistry, vol. 48, no. 1, pp. 21-26, 2010.

[26] X. Ou, Y. Zhang, C. Xu et al., “Transgenerational inheritance of modified DNA methylation patterns and enhanced tolerance induced by heavy metal stress in rice (Oryza sativa L.)," PLoS ONE, vol. 7, no. 9, Article ID e41143, 2012.

[27] H. P. Kou, Y. Li, X. X. Song et al., "Heritable alteration in DNA methylation induced by nitrogen-deficiency stress accompanies enhanced tolerance by progenies to the stress in rice (Oryza sativa L.)," Journal of Plant Physiology, vol. 168, no. 14, pp. 16851693, 2011.

[28] M. M. Suzuki and A. Bird, "DNA methylation landscapes: provocative insights from epigenomics," Nature Reviews Genetics, vol. 9, no. 6, pp. 465-476, 2008.

[29] R. S. Sekhon and S. Chopra, "Progressive loss of DNA methylation releases epigenetic gene silencing from a tandemly repeated maize Myb gene," Genetics, vol. 181, no. 1, pp. 81-91, 2009.

[30] M. Kato, A. Miura, J. Bender, S. E. Jacobsen, and T. Kakutani, "Role of CG and non-CG methylation in immobilization of transposons in Arabidopsis," Current Biology, vol. 13, no. 5, pp. 421-426, 2003.

[31] S. Hashida, K. Kitamura, T. Mikami, and Y. Kishima, “Temperature shift coordinately changes the activity and the methylation state of transposon Tam3 in Antirrhinum majus," Plant Physiology, vol. 132, no. 3, pp. 1207-1216, 2003.

[32] C. Cheng, M. Daigen, and H. Hirochika, "Epigenetic regulation of the rice retrotransposon Tos17," Molecular Genetics and Genomics, vol. 276, no. 4, pp. 378-390, 2006.

[33] J. C. Rice and C. D. Allis, "Histone methylation versus histone acetylation: new insights into epigenetic regulation," Current Opinion in Cell Biology, vol. 13, no. 3, pp. 263-273, 2001.

[34] S. L. Berger, "The complex language of chromatin regulation during transcription," Nature, vol. 447, no. 7143, pp. 407-412, 2007.

[35] J.-M. Kim, T. K. To, J. Ishida et al., "Alterations of lysine modifications on the histone $\mathrm{H} 3 \mathrm{~N}$-tail under drought stress conditions in Arabidopsis thaliana," Plant \& Cell Physiology, vol. 49, no. 10, pp. 1580-1588, 2008.

[36] E. J. Stockinger, Y. Mao, M. K. Regier, S. J. Triezenberg, and M. F. Thomashow, "Transcriptional adaptor and histone acetyltransferase proteins in Arabidopsis and their interactions with $\mathrm{CBF} 1$, a transcriptional activator involved in cold-regulated gene expression," Nucleic Acids Research, vol. 29, no. 7, pp. 15241533, 2001.

[37] Z. J. Chen and L. Tian, "Roles of dynamic and reversible histone acetylation in plant development and polyploidy," Biochimica et Biophysica Acta, vol. 1769, no. 5-6, pp. 295-307, 2007. 
[38] C. Hollender and Z. Liu, "Histone deacetylase genes in Arabidopsis development," Journal of Integrative Plant Biology, vol. 50, no. 7, pp. 875-885, 2008.

[39] F. Pontvianne, T. Blevins, and C. S. Pikaard, "Arabidopsis histone lysine methyltransferases," Advances in Botanical Research, vol. 53, pp. 1-22, 2010.

[40] X. Yu, L. Li, M. Guo, J. Chory, and Y. Yin, "Modulation of brassinosteroid-regulated gene expression by jumonji domaincontaining proteins ELF6 and REF6 in Arabidopsis," PNAS, vol. 105, no. 21, pp. 7618-7623, 2008.

[41] A. Miura, M. Nakamura, S. Inagaki, A. Kobayashi, H. Saze, and T. Kakutani, "An Arabidopsis jmjC domain protein protects transcribed genes from DNA methylation at CHG sites," EMBO Journal, vol. 28, no. 8, pp. 1078-1086, 2009.

[42] D. Vermaak, K. Ahmad, and S. Henikoff, "Maintenance of chromatin states: an open-and-shut case," Current Opinion in Cell Biology, vol. 15, no. 3, pp. 266-274, 2003.

[43] D. M. Bond, E. S. Dennis, B. J. Pogson, and E. J. Finnegan, "Histone acetylation, vernalization insensitive 3 , flowering locus $\mathrm{C}$, and the vernalization response," Molecular Plant, vol. 2, no. 4, pp. 724-737, 2009.

[44] J. Zhu, J. C. Jae, Y. Zhu et al., "Involvement of Arabidopsis HOS15 in histone deacetylation and cold tolerance," Proceedings of the National Academy of Sciences of the United States of America, vol. 105, no. 12, pp. 4945-4950, 2008.

[45] S. Sridha and K. Wu, "Identification of AtHD2C as a novel regulator of abscisic acid responses in Arabidopsis," Plant Journal, vol. 46, no. 1, pp. 124-133, 2006.

[46] A. Gendrel, Z. Lippman, C. Yordan, V. Colot, and R. A. Martienssen, "Dependence of heterochromatic histone H3 methylation patterns on the Arabidopsis gene DDM1," Science, vol. 297, no. 5588, pp. 1871-1873, 2002.

[47] L. M. Johnson, X. Cao, and S. E. Jacobsen, "Interplay between two epigenetic marks: DNA methylation and histone $\mathrm{H} 3$ lysine 9 methylation," Current Biology, vol. 12, no. 16, pp. 1360-1367, 2002.

[48] K. Zhang, V. V. Sridhar, J. Zhu, and A. Kapoor, "Distinctive core histone post-translational modification patterns in Arabidopsis thaliana," PLoS ONE, vol. 2, no. 11, Article ID e1210, 2007.

[49] F. Turck, F. Roudier, S. Farrona et al., "Arabidopsis TFL2/LHP1 specifically associates with genes marked by trimethylation of histone H3 lysine 27," PLoS Genetics, vol. 3, article e86, 2007.

[50] Y. V. Bernatavichute, X. Zhang, S. Cokus, M. Pellegrini, and S. E. Jacobsen, "Genome-wide association of histone H3 lysine nine methylation with CHG DNA methylation in Arabidopsis thaliana," PLoS ONE, vol. 3, no. 9, Article ID e3156, 2008.

[51] X. Zhang, Y. V. Bernatavichute, S. Cokus, M. Pellegrini, and S. E. Jacobsen, "Genome-wide analysis of mono-, di- and trimethylation of histone $\mathrm{H} 3$ lysine 4 in Arabidopsis thaliana," Genome Biology, vol. 10, no. 6, article R62, 2009.

[52] A. Boyko, P. Kathiria, F. J. Zemp, Y. Yao, I. Pogribny, and I. Kovalchuk, "Transgenerational changes in the genome stability and methylation in pathogen-infected plants: (Virus-induced plant genome instability)," Nucleic Acids Research, vol. 35, no. 5, pp. 1714-1725, 2007.

[53] G. S. Scippa, M. di Michele, E. Onelli, G. Patrignani, D. Chiatante, and E. A. Bray, "The histone-like protein H1-S and the response of tomato leaves to water deficit," Journal of Experimental Botany, vol. 55, no. 394, pp. 99-109, 2004.

[54] H. Tsuji, H. Saika, N. Tsutsumi, A. Hirai, and M. Nakazono, "Dynamic and reversible changes in histone H3-Lys4 methylation and $\mathrm{H} 3$ acetylation occurring at submergence-inducible genes in rice," Plant and Cell Physiology, vol. 47, no. 7, pp. 9951003,2006

[55] K. van Dijk, Y. Ding, S. Malkaram et al., "Dynamic changes in genome-wide histone $\mathrm{H} 3$ lysine 4 methylation patterns in response to dehydration stress in Arabidopsis thaliana," BMC Plant Biology, vol. 10, article 238, 2010.

[56] A. Sokol, A. Kwiatkowska, A. Jerzmanowski, and M. Prymakowska-Bosak, "Up-regulation of stress-inducible genes in tobacco and Arabidopsis cells in response to abiotic stresses and $\mathrm{ABA}$ treatment correlates with dynamic changes in histone $\mathrm{H} 3$ and $\mathrm{H} 4$ modifications," Planta, vol. 227, no. 1, pp. 245-254, 2007.

[57] Z. Zhang, S. Zhang, Y. Zhang et al., “Arabidopsis floral initiator SKB1 confers high salt tolerance by regulating transcription and pre-mRNA splicing through altering histone H4R3 and small nuclear ribonucleoprotein LSM4 methylation," Plant Cell, vol. 23, no. 1, pp. 396-411, 2011.

[58] R. Sunkar, V. Chinnusamy, J. Zhu, and J. Zhu, "Small RNAs as big players in plant abiotic stress responses and nutrient deprivation," Trends in Plant Science, vol. 12, no. 7, pp. 301-309, 2007.

[59] T. A. Volpe, C. Kidner, I. M. Hall, G. Teng, S. I. S. Grewal, and R. A. Martienssen, "Regulation of heterochromatic silencing and histone H3 lysine-9 methylation by RNAi," Science, vol. 297, no. 5588, pp. 1833-1837, 2002.

[60] J. R. Tuttle, A. M. Idris, J. K. Brown, C. H. Haigler, and D. Robertson, "Geminivirus-mediated gene silencing from cotton leaf crumple virus is enhanced by low temperature in cotton," Plant Physiology, vol. 148, no. 1, pp. 41-50, 2008.

[61] Y. Okano, D. Miki, and K. Shimamoto, "Small interfering RNA (siRNA) targeting of endogenous promoters induces DNA methylation, but not necessarily gene silencing, in rice," Plant Journal, vol. 53, no. 1, pp. 65-77, 2008.

[62] B. J. Reinhart, E. G. Weinstein, M. W. Rhoades, B. Bartel, and D. P. Bartel, "MicroRNAs in plants," Genes and Development, vol. 16, no. 13, pp. 1616-1626, 2002.

[63] P. Gao, X. Bai, L. Yang et al., "Over-expression of osa-MIR396c decreases salt and alkali stress tolerance," Planta, vol. 231, no. 5, pp. 991-1001, 2010.

[64] R. Sunkar and J. K. Zhu, "Novel and stress regulated microRNAs and other small RNAs from Arabidopsis," Plant Cell, vol. 16, no. 8, pp. 2001-2019, 2004.

[65] J. Zhang, Y. Xu, Q. Huan, and K. Chong, "Deep sequencing of Brachypodium small RNAs at the global genome level identifies microRNAs involved in cold stress response," BMC Genomics, vol. 10, article 1471, p. 449, 2009.

[66] D. K. Lv, X. Bai, Y. Li et al., "Profiling of cold-stress-responsive miRNAs in rice by microarrays," Gene, vol. 459, no. 1-2, pp. 3947, 2010.

[67] R. Sunkar, A. Kapoor, and J. Zhu, "Posttranscriptional induction of two $\mathrm{Cu} / \mathrm{Zn}$ superoxide dismutase genes in Arabidopsis is mediated by downregulation of miR398 and important for oxidative stress tolerance," Plant Cell, vol. 18, no. 8, pp. 20512065, 2006.

[68] H. S. Guo, Q. Xie, J. F. Fei, and N. H. Chua, "MicroRNA directs mRNA cleavage of the transcription factor NAC1 to downregulate auxin signals for Arabidopsis lateral root development," Plant Cell, vol. 17, no. 5, pp. 1376-1386, 2005.

[69] L. Navarro, P. Dunoyer, F. Jay et al., "A plant miRNA contributes to antibacterial resistance by repressing auxin signaling," Science, vol. 312, no. 5772, pp. 436-439, 2006. 
[70] A. Verdel, S. Jia, S. Gerber et al., "RNAi-mediated targeting of heterochromatin by the RITS complex," Science, vol. 303, no. 5658, pp. 672-676, 2004.

[71] S. I. S. Grewal and D. Moazed, "Heterochromatin and epigenetic control of gene expression," Science, vol. 301, no. 5634, pp. 798802, 2003.

[72] Y. Yao, A. Bilichak, A. Golubov, T. Blevins, and I. Kovalchuk, "Differential sensitivity of Arabidopsis siRNA biogenesis mutants to genotoxic stress," Plant Cell Reports, vol. 29, no. 12, pp. 1401-1410, 2010.

[73] O. Borsani, J. Zhu, P. E. Verslues, and R. Sunkar, "Endogenous siRNAs derived from a pair of natural cis-antisense transcripts regulate salt tolerance in Arabidopsis," Cell, vol. 123, no. 7, pp. 1279-1291, 2005.

[74] M. E. Alvarez, F. Nota, and D. A. Cambiagno, "Epigenetic control of plant immunity," Molecular Plant Pathology, vol. 11, no. 4, pp. 563-576, 2010.

[75] P. Sudarsanam and F. Winston, "The Swi/Snf family: nucleosome-remodeling complexes and transcriptional control," Trends in Genetics, vol. 16, no. 8, pp. 345-351, 2000.

[76] L. Mlynárová, J. Nap, and T. Bisseling, “The SWI/SNF chromatin-remodeling gene AtCHR12 mediates temporary growth arrest in Arabidopsis thaliana upon perceiving environmental stress," Plant Journal, vol. 51, no. 5, pp. 874-885, 2007.

[77] A. Saez, A. Rodrigues, J. Santiago, S. Rubio, and P. L. Rodriguez, "HAB1-SWI3B interaction reveals a link between abscisic acid signaling and putative SWI/SNF chromatin-remodeling complexes in Arabidopsis," Plant Cell, vol. 20, no. 11, pp. 2972-2988, 2008.

[78] E. Perruc, N. Kinoshita, and L. Lopez-Molina, "The role of chromatin-remodeling factor PKL in balancing osmotic stress responses during Arabidopsis seed germination," Plant Journal, vol. 52, no. 5, pp. 927-936, 2007.

[79] A. Dong, Z. Liu, Y. Zhu et al., "Interacting proteins and differences in nuclear transport reveal specific functions for the NAP1 family proteins in plants," Plant Physiology, vol. 138, no. 3, pp. 1446-1456, 2005.

[80] Z. Q. Liu, J. Gao, A. W. Dong, and W. H. Shen, "A truncated Arabidopsis nucleosome assembly protein 1, AtNAP1;3T , alters plant growth responses to abscisic acid and salt in the Atnapl ; 3-2 mutant," Molecular Plant, vol. 2, no. 4, pp. 688-699, 2009.

[81] L. Hennig, R. Bouveret, and W. Gruissem, "MSI1-like proteins: an escort service for chromatin assembly and remodeling complexes," Trends in Cell Biology, vol. 15, no. 6, pp. 295-302, 2005.

[82] C. Alexandre, Y. Möller-Steinbach, N. Schönrock, W. Gruissem, and L. Hennig, "Arabidopsis MSI1 is required for negative regulation of the response to drought stress," Molecular Plant, vol. 2, no. 4, pp. 675-687, 2009.

[83] H. Kim, Y. Hyun, J. Park et al., "A genetic link between cold responses and flowering time through FVE in Arabidopsis thaliana," Nature Genetics, vol. 36, no. 2, pp. 167-171, 2004.

[84] H. H. Q. Heng, S. W. Bremer, J. B. Stevens, K. J. Ye, G. Liu, and C. J. Ye, "Genetic and epigenetic heterogeneity in cancer: a genome-centric perspective," Journal of Cellular Physiology, vol. 220, no. 3, pp. 538-547, 2009. 

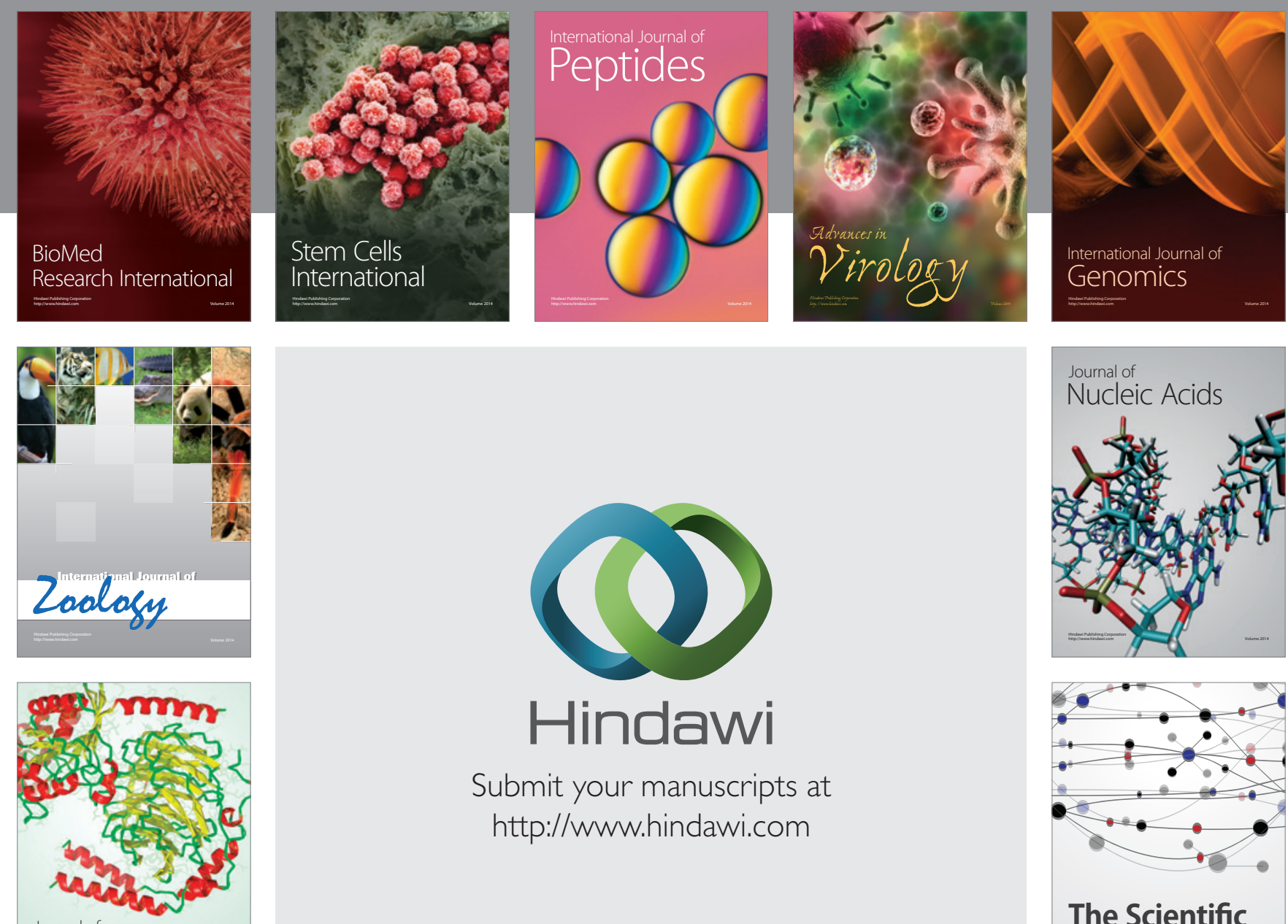

Submit your manuscripts at

http://www.hindawi.com

Journal of
Signal Transduction
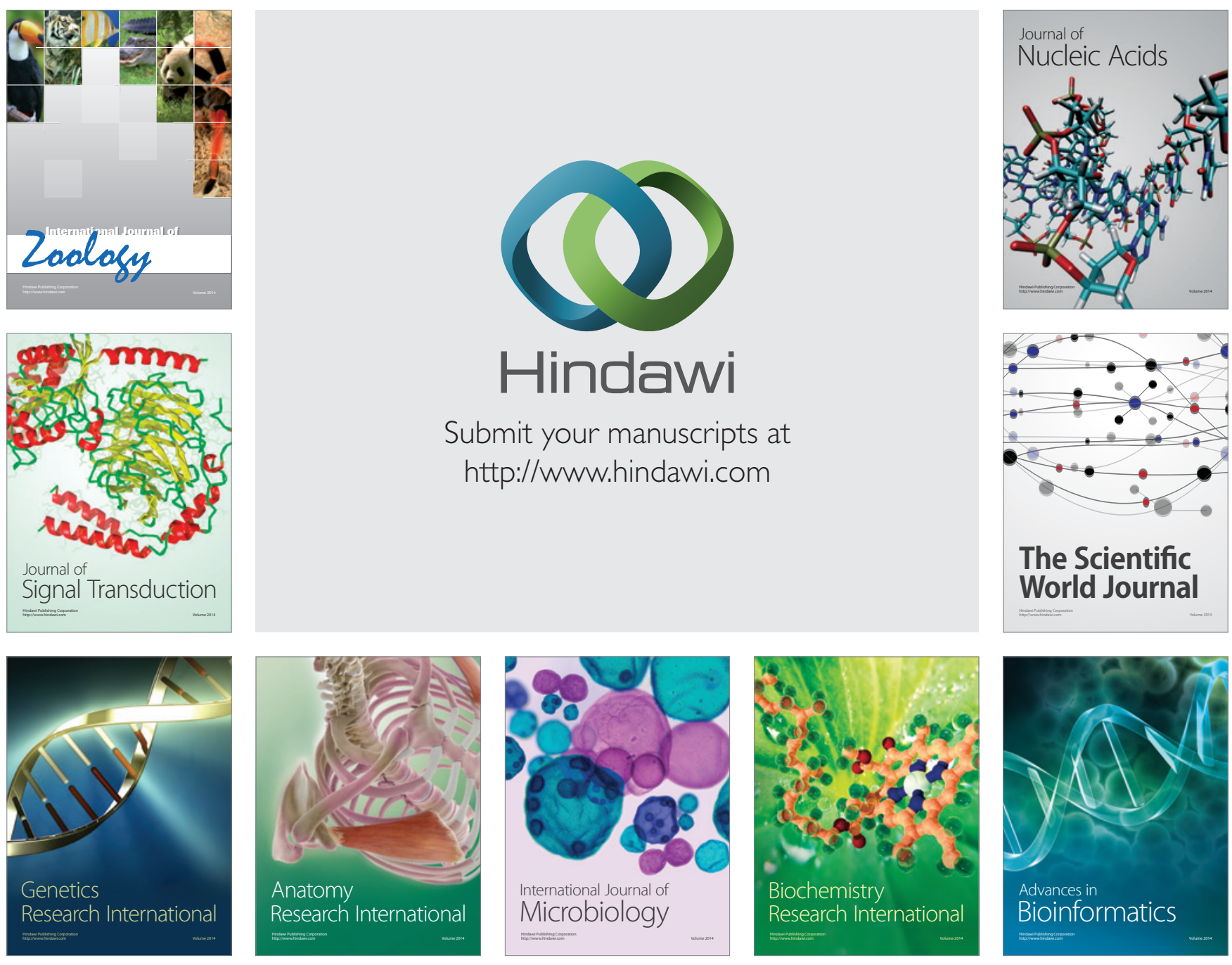

The Scientific World Journal
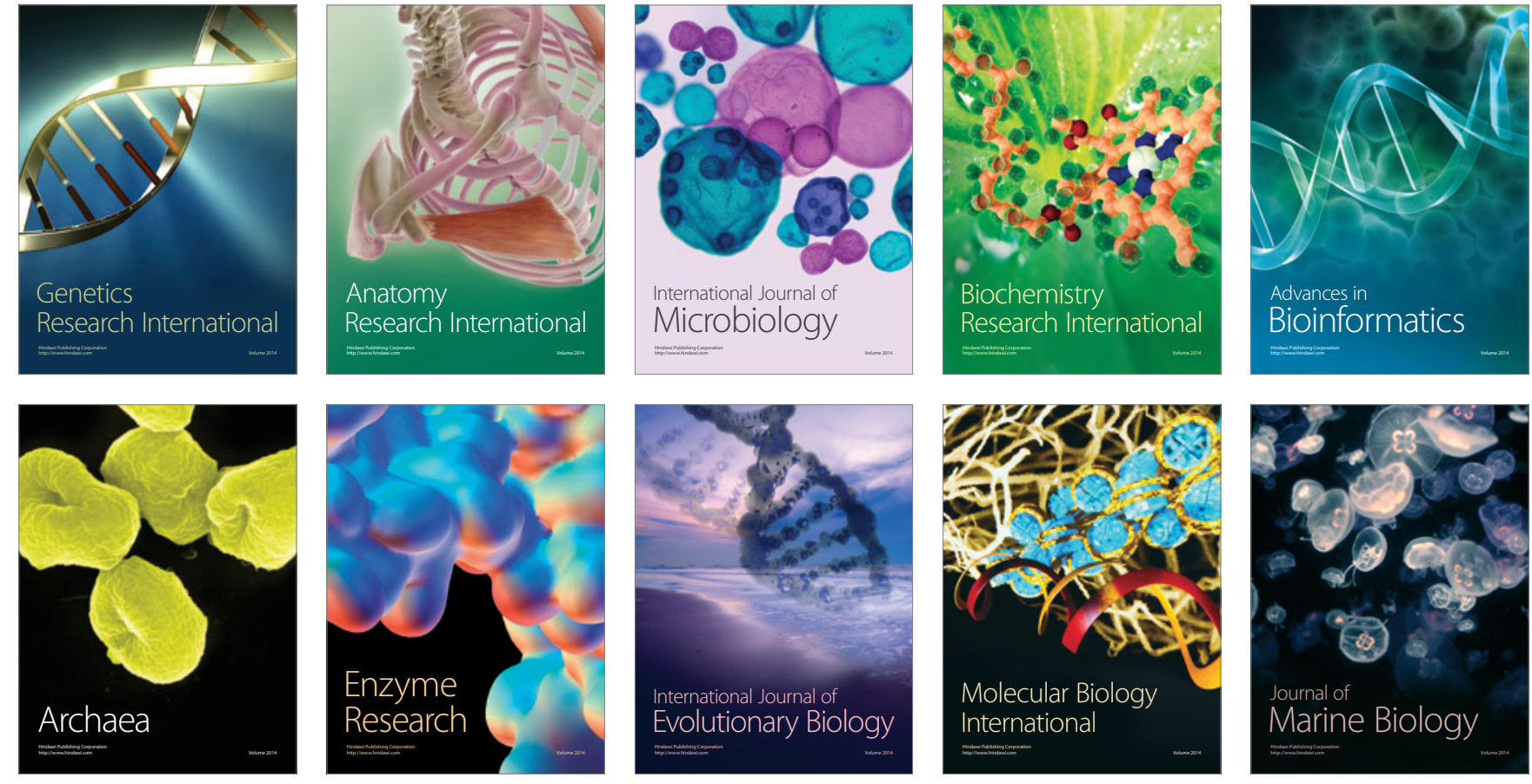